\title{
Longest Induced Cycles in Circulant Graphs
}

\author{
Elena D. Fuchs \\ Department of Mathematics \\ University of California, Berkeley, Berkeley, CA \\ lenfuchs@berkeley.edu
}

Submitted: Aug 19, 2004; Accepted: Jun 13, 2005; Published: Oct 13, 2005

Mathematics Subject Classifications: 05C88, 05C89

\begin{abstract}
In this paper we study the length of the longest induced cycle in the unit circulant graph $X_{n}=\operatorname{Cay}\left(\mathbb{Z}_{n} ; \mathbb{Z}_{n}^{*}\right)$, where $\mathbb{Z}_{n}^{*}$ is the group of units in $\mathbb{Z}_{n}$. Using residues modulo the primes dividing $n$, we introduce a representation of the vertices that reduces the problem to a purely combinatorial question of comparing strings of symbols. This representation allows us to prove that the multiplicity of each prime dividing $n$, and even the value of each prime (if sufficiently large) has no effect on the length of the longest induced cycle in $X_{n}$. We also see that if $n$ has $r$ distinct prime divisors, $X_{n}$ always contains an induced cycle of length $2^{r}+2$, improving the $r \ln r$ lower bound of Berrezbeitia and Giudici. Moreover, we extend our results for $X_{n}$ to conjunctions of complete $k_{i}$-partite graphs, where $k_{i}$ need not be finite, and also to unit circulant graphs on any quotient of a Dedekind domain.
\end{abstract}

\section{Introduction}

For a positive integer $n$, let the unit circulant graph $X_{n}=\operatorname{Cay}\left(\mathbb{Z}_{n}, \mathbb{Z}_{n}^{*}\right)$ be defined as follows:

(1) The vertex set of $X_{n}$, denoted by $V(n)$, is $\mathbb{Z}_{n}$, the ring of integers modulo $n$.

(2) The edge set of $X_{n}$ is denoted by $E(n)$, and, for $x, y \in V(n),\{x, y\} \in E(n)$ if and only if $x-y \in \mathbb{Z}_{n}^{*}$, where $\mathbb{Z}_{n}^{*}$ is the set of units in the ring $\mathbb{Z}_{n}$.

The central problem adressed in this paper is to find the length of the longest induced cycle in $X_{n}$. This problem was first considered by Berrizbeitia and Giudici [1], who were motivated by its applications to chromatic uniqueness.

Throughout the paper, we let $n=p_{1}^{a_{1}} p_{2}^{a_{2}} \ldots p_{r}^{a_{r}}$, where the $p_{i}$ are distinct primes, and $a_{i} \geq 1$. Then we denote the length of the longest induced cycle in $X_{n}$ by $m(n)$. We let $M(r)=\max _{n} m(n)$, where the maximum is taken over all $n$ with $r$ distinct prime divisors. In [1], Berrizbeitia and Giudici bound $M(r)$ by

$$
r \ln r \leq M(r) \leq 9 r !
$$


A simple change to the proof of the upper bound provided in [1] yields the better upper bound of $M(r) \leq 6 r !$.

Our goal is to determine better bounds for $M(r)$, as well as to extend what we find to other graphs. In Section 2, we introduce a useful representation of the vertices in $X_{n}$ according to their residues modulo the prime divisors of $n$. This representation immediately yields several helpful properties of the longest induced cycles in these graphs. In particular, we prove that we can disregard the multiplicities of the prime divisors of $n$, so we can reduce our problem to square-free $n$. Also, we show that $m(n)$ depends only on $r$, and in fact $m(n)=M(r)$ as long as the primes dividing $n$ are all large enough. In Section 3, we use the vertex representation introduced in Section 2 to construct an induced cycle of length $2^{r}+2$ in the graph $X_{n}$, where $n$ has $r$ distinct prime divisors, thus raising the lower bound on $M(r)$ substantially. We also note that this construction is valid for any $n$, no matter what its prime divisors are, so this provides a lower bound for $m(n)$. Section 4 contains a generalization of our results to conjunctions of complete $k_{i}$-partite graphs, as well as to unit circulant graphs on products of local rings, which include the unit circulant graphs on Dedekind rings.

\section{Residue Representation}

Recall that $n=p_{1}^{a_{1}} p_{2}^{a_{2}} \cdots p_{r}^{a_{r}}$, where the $p_{i}$ are prime. We will represent the vertices of $X_{n}$ in a way that will reduce the process of finding induced cycles in $X_{n}$ to checking for similarities between strings of numbers in an array.

It is clear that the following is equivalent to the definition of $E(n)$ in the introduction:

Observation 2.1. For $x, y \in V(n)$, we have that $\{x, y\} \in E(n)$ if and only if

$$
x \not \equiv y \quad\left(\bmod p_{i}\right), \text { for all } 1 \leq i \leq r .
$$

Likewise, $\{x, y\} \notin E(n)$ if and only if

$$
x \equiv y \quad\left(\bmod p_{i}\right), \text { for some } 1 \leq i \leq r .
$$

So, in fact, to know whether $x$ and $y$ are adjacent we need only their residues modulo the primes $p_{i}$. With this in mind, we introduce the following representation of the vertices:

\section{Definition 2.2.}

(i) Let $x \in V(n)$, such that

$$
x \equiv \alpha_{i} \quad\left(\bmod p_{i}\right), \text { where } 1 \leq i \leq r \text { and } 0 \leq \alpha_{i}<p_{i} .
$$

We then define the residue representation of $x$ to be the unique string $\alpha_{1} \alpha_{2} \cdots \alpha_{r}$, where $\alpha_{k}$ is the $k$ th term, and we write $x \approx \alpha_{1} \alpha_{2} \cdots \alpha_{r}$.

(ii) Let $x, y \in V(n)$. If the $k$ th term of the residue representation of $x$ is the same as the $k$ th term of the residue representation of $y$, we say that $x$ has a coincidence with $y$. 
Combining Observation 2.1 and Definition 2.2, vertices $x, y \in V(n)$ are adjacent if and only if $x$ has no coicidences with $y$. So, in fact, the only property of the residues modulo $p_{i}$ that we use in constructing induced cycles is that they form a set of size $p_{i}$, and we verify that a subgraph is an induced cycle by checking that consecutive vertices do not have any coincidences, and that any pair of non-consecutive vertices has at least one coincidence.

Also, we note that for $n$ not square-free, a string may be the residue representation of multiple vertices. For example, if $n=12$, both 0 and 6 have residue representation 00 . However, the adjacency of vertices depends only on their residue representations, and, by the Chinese Remainder Theorem, every string represents at least one vertex.

This representation greatly simplifies inspection of induced cycles. In fact, we can extend residue representation for a vertex to any induced subgraph:

\section{Definition 2.3.}

(i) Let $S$ be an induced subgraph of $X_{n}$, where $V(S)=\left(v_{0}, v_{2}, \ldots, v_{k-1}\right)$, with $v_{i} \approx$ $\alpha_{i 1} \alpha_{i 2} \cdots \alpha_{i r}$, and $0 \leq i \leq k-1$. We then define the residue representation of $S$ to be the array

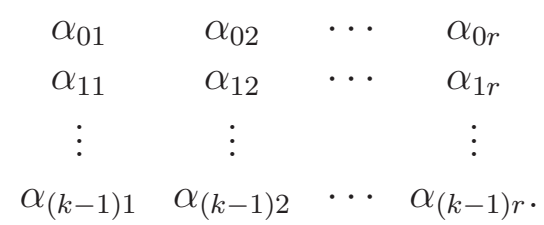

(ii) The residue set of $S$ is the set of residues

$$
\left\{\alpha_{i j} \mid 0 \leq i \leq k-1,1 \leq j \leq r\right\}
$$

used in its residue representation.

So, if an induced subgraph $S$ is a $k$-cycle in $X_{n}$, we can permute the rows of the residue representation of $S$ so that the $i$ th row has a coincidence with the $j$ th row if and only if $i-j \not \equiv \pm 1(\bmod k)$. Figure 1 displays the residue representation of an induced 6-cycle for $r=2$ and for $r=3$.

An important property of an induced cycle of length greater than 4 is that it cannot contain two vertices with the same residue representation.

Proposition 2.4. The residue representation of a $k$-cycle $C$, with $k>4$, cannot contain two identical rows.

Proof. Suppose there are two vertices $x$ and $y$ in $C$ that have the same residue representation. Then a vertex $z$ of $C$ has no coincidence with $x$ if and only if it has no coincidence with $y$, meaning that $x$ and $y$ have precisely the same neighbors in $C$. However, a vertex in an induced cycle is adjacent to exactly two other vertices in the cycle, so $C$ can have at most 4 vertices, contradicting $k>4$. Thus the residue representation of $C$ cannot contain two identical rows. 


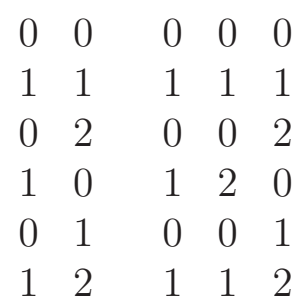

Figure 1: In these residue representations of an induced 6-cycle for $r=2$ on the left, and for $r=3$ on the right, it is easy to see that two consecutive rows (including the 1st and 6th rows) have no coincidences, and any two non-consecutive rows have at least one coincidence. The residue set for each cycle is $\{0,1,2\}$.

It is important that, once we have written an induced cycle in terms of its residue representation, we can permute the residues in each column to obtain an induced cycle of equal length.

Observation 2.5. Let the $j$ th column in the residue representation of an induced $k$-cycle $C$ in $X_{n}$ be

$$
\begin{gathered}
\alpha_{0 j} \\
\alpha_{1 j} \\
\vdots \\
\alpha_{(k-1) j},
\end{gathered}
$$

and suppose this column contains $l_{j}$ distinct residues, $\left\{s_{1}, s_{2}, \ldots, s_{l_{j}}\right\}$. Then let $\pi$ be a permutation of $\left\{s_{1}, s_{2}, \ldots, s_{l_{j}}\right\}$, and replace the $j$ th column of $C$ by

$$
\begin{gathered}
\pi\left(\alpha_{0 j}\right) \\
\pi\left(\alpha_{1 j}\right) \\
\vdots \\
\pi\left(\alpha_{(k-1) j}\right) .
\end{gathered}
$$

We then have a new induced $k$-cycle in $X_{n}$, since we have not changed the coincidences between any of the rows in $C$.

We now use the Observation 2.5 to define isomorphisms between induced $k$-cycles in $X_{n}$.

Definition 2.6. Two induced $k$-cycles, $C$ and $C^{\prime}$, are called isomorphic if, for every $j$, the $j$ th column of the residue representation of $C^{\prime}$ is obtained by permuting the residues in the $j$ th column of $C$, as described in Observation 2.5.

Note that the first two rows in Figure 1 are 000 and 111. Because of this, all of the rows that are not adjacent to either of the first two have to contain both a 0 and a 1 . Similarly, the third row in the cycle must contain a 0 , and the last row in the cycle must contain a 1. This is a useful criterion for induced cycles in general. 
Remark 2.7. Any induced cycle $C$ in $X_{n}$ is isomorphic to an induced cycle $C^{\prime}$ of the same length so that the first two rows in the residue representation of $C^{\prime}$ are $00 \cdots 0$ and $11 \cdots 1$.

In order to obtain such a $C^{\prime}$, we need only to map the first two elements in every column of $C$ to 0 and 1, respectively. Note that the first two elements in each column are always different - if they were not, the first and the second row in the residue representation of $C$ would have a coincidence, which contradicts their adjacency.

This tells us that all but four of the rows in our induced cycles will have to contain both a 0 and a 1 , which may limit the residue sets and consequently the lengths of the cycles.

Another interesting fact that becomes evident with the use of residue representation is the following proposition.

Proposition 2.8. The value $M(r)$ increases with $r$. Specifically, if $X_{n}$ contains an induced cycle of length $k$, and $q>2$ is a prime not dividing $n$, then $X_{q n}$ also contains a cycle of length $k$. If $k$ is even, we can also allow $q=2$.

Proof. Let $n=p_{1}^{a_{1}} p_{2}^{a_{2}} \cdots p_{r}^{a_{r}}$, where the exponents $a_{i}$ are positive integers, and $p_{i}$ are distinct primes. Suppose $X_{n}$ contains an induced cycle $C$ of length $k$. We denote the residue representations of the vertices of $C$ by $v_{0}, v_{1}, \ldots, v_{k-1}$, where each $v_{i}$ is a string of length $r$. Let $n^{\prime}=q n$, where $q \neq 2$ is prime, $q \neq p_{i}$ for all $1 \leq i \leq r$. Then we will show that $X_{n^{\prime}}$ also contains a cycle of length $k$ by constructing an induced cycle $C^{\prime}$ in $X_{n^{\prime}}$, denoting the residue representations of the vertices of $C^{\prime}$ by $w_{0}, w_{1}, \ldots, w_{k-1}$.

If $k$ is even, let $w_{i}=v_{i} 0$ for even $i$, and let $w_{i}=v_{i} 1$ for odd $i$. Notice that we do not introduce any coincidences between two rows that were adjacent in $C$, so two consecutive rows in $C^{\prime}$ are adjacent, as desired. Similarly, if $\left\{v_{i}, v_{j}\right\} \notin E(n)$, they have a coincidence, say, in the $l$ th term. Then $w_{i}$ and $w_{j}$ have a coincidence in the $l$ th term, and so $\left\{w_{i}, w_{j}\right\} \notin E\left(n^{\prime}\right)$. Thus we introduce no new adjacencies in the construction of $C^{\prime}$, so $C^{\prime}$ is indeed an induced $k$-cycle in $X_{n^{\prime}}$.

If $k$ is odd, let $w_{i}=v_{i} 1$ for odd $i$, let $w_{i}=v_{i} 0$ for even $i \neq k-1$, and let $w_{k-1}=v_{k-1} 2$ (this is possible since $q \neq 2$ ). Again, we note that we do not introduce any coincidences between two rows that were adjacent in $C$, so two consecutive rows in $C^{\prime}$ are adjacent, as desired. Also, if $\left\{v_{i}, v_{j}\right\} \notin E(n)$, we have that $\left\{w_{i}, w_{j}\right\} \notin E\left(n^{\prime}\right)$ by the argument above. Thus we introduce no new adjacencies in the construction of $C^{\prime}$, so $C^{\prime}$ is indeed an induced $k$-cycle in $X_{n^{\prime}}$.

By starting with a cycle $C$ in $X(n)$ that has length $M(r)$, we see that $M(r+1) \geq M(r)$, as desired.

Corollary 2.9. If $r \geq 2$, and $n$ is square-free, then $m(n) \geq 6$.

Proof. For $r=2$, we have constructed a 2-cycle of length 6 in Figure 1, so $M(2) \geq 6$. Proposition 2.8 shows that $M(r)$ is nondecreasing, so we have that, if $r>2, M(r) \geq$ $M(2) \geq 6$, as desired.

We now prove that, in calculating $m(n)$, we need consider only those $n$ that are square-free. 
Theorem 2.10. For $n=p_{1}^{a_{1}} p_{2}^{a_{2}} \cdots p_{r}^{a_{r}}$, and $n^{\prime}=p_{1} p_{2} \cdots p_{r}$, where $r \neq 1, m(n)=M\left(n^{\prime}\right)$.

Proof. (1) First we show that $m(n) \geq M\left(n^{\prime}\right)$. In particular, we show $X_{n}$ contains cycles of length $M\left(n^{\prime}\right)$. Note that since $n$ and $n^{\prime}$ have the same prime divisors, if $x, y<n$, then $x-y \in \mathbb{Z}_{n}^{*}$ if and only if $x-y \in \mathbb{Z}_{n^{\prime}}^{*}$. So, in particular, the induced subgraph of $X_{n}$ on vertices $0,1, \ldots, n^{\prime}-1$ is precisely $X_{n^{\prime}}$. Thus any induced cycle on $X_{n^{\prime}}$ can be mapped to an induced cycle in $\left\{0,1, \ldots, n^{\prime}-1\right\} \subset X_{n}$, and so there is an induced cycle of length $M\left(n^{\prime}\right)$ in $X_{n}$, as desired.

(2) Now we show that $m(n) \leq M\left(n^{\prime}\right)$, or that there is no induced cycle of length greater than $M\left(n^{\prime}\right)$ in $X_{n}$. Since $n^{\prime}$ is square-free, Corollary 2.9 implies that $M\left(n^{\prime}\right) \geq 6$. Suppose there is an induced cycle, $C_{l}$, of length $l>M\left(n^{\prime}\right)$ in $X_{n}$. Then, in particular, $l>6$. Using residue representation, write $C_{l}$ in terms of residues $\left(\bmod p_{1}, p_{2}, \ldots, p_{r}\right)$. If no two vertices in $C_{l}$ are denoted by the same string of residues, then we can view the residue representation of $C_{l}$ as a residue representation of an induced $l$-cycle in $X_{n^{\prime}}$. Since $l>M\left(n^{\prime}\right)$, this contradicts the assumption that $M\left(n^{\prime}\right)$ is the maximum length of an induced cycle in $X_{n^{\prime}}$. Thus there exist two vertices in $C_{l}$ that have identical residue representations. However, by Proposition 2.4, this means $l \leq 4$, contradicting the previous deduction that that $l>6$. We conclude that, indeed, there are no induced cycles of length $l>M\left(n^{\prime}\right)$ in $X_{n}$.

Proposition 2.11. Let $n^{\prime}=p$, and $n=p^{a}$ where $p$ is a prime and $a>1$. Then $M\left(n^{\prime}\right)=3$, and $m(n)=4$. So, $M(1)=4$.

Proof. Since the only non-unit in $\mathbb{Z}_{p}$ is $0, X_{n^{\prime}}$ is a complete graph on $p$ vertices, and the longest induced cycle in $X_{n^{\prime}}$ must hence have length 3. From Part (2) of the proof of Theorem 2.10, we deduce that $m(n) \leq 4$. In fact, $m(n)=4$, since the subgraph $(0,1, p, p+1)$ is an induced cycle in $X_{n}$.

Proposition 2.12. For $n=p_{1}^{a_{1}} p_{2}^{a_{2}} \cdots p_{r}^{a_{r}}$ where the $p_{i}$ are large, $m(n)=M(r)$.

Proof. Let $n^{\prime}$ be a positive integer with exactly $r$ prime divisors, such that $m\left(n^{\prime}\right)=M(r)$, and let $C$ be a longest induced cycle in $X_{n^{\prime}}$. Assume each $p_{i}$ is larger than the number of residues that appear in the residue representation of $C$. Then there is a subgraph $S$ of $X_{n}$, such that the residue representation of $S$ is the same as the residue representation of $C$. So $S$ is an induced cycle of length $M(r)$. Hence $m(n)=M(r)$. Thus, as long as the prime divisors of $n$ yield enough residues for a residue representation of the longest cycle in $X_{n^{\prime}}$, where $M\left(n^{\prime}\right)=M(r)$, we will have $m(n)=M(r)$.

\section{A Lower Bound on $M(r)$}

One important asset of introducing residue representation is that it gives us a way to construct a good lower bound on $M(r)$; we achieve the following lower bound as our main result in this section.

Theorem 3.1. For all positive integers $n$ with $r>1$ distinct prime divisors, we have $M(r) \geq 2^{r}+2$. 
In this section, we construct an induced subgraph of $X_{n}$ with $2^{r}+2$ vertices, where $r$ is the number of distinct prime divisors of $n$, and provide two specific cycles produced by this construction. We will then prove that this subgraph is indeed a cycle, and thus show that Theorem 3.1 holds.

In order to construct an induced $2^{r}+2$-cycle in $X_{n}$, where $n=p_{1} p_{2} \cdots p_{r}$, we first introduce some definitions, which are discussed in detail in [4], p. 433.

(i) An $n$-bit Gray Code is an ordered, cyclic sequence of the $2^{n} n$-bit binary strings called codewords, such that successive codewords differ by the complementation of a single bit, and the starting codeword is taken to be $(00 \cdots 0)$. We write this sequence in the form of a matrix, as shown below.

(ii) A Reflective Gray Code (RGC) is defined recursively as follows: A 1-bit RGC is merely the $2 \times 1$ matrix $\left(\begin{array}{l}0 \\ 1\end{array}\right)$. If an $r$-bit RGC is the $2^{r} \times r$ binary matrix

$$
\left(\begin{array}{l}
G_{0} \\
G_{1} \\
\vdots \\
G_{2^{r}-1},
\end{array}\right)
$$

then we define the $(r+1)$-bit RGC to be the $2^{r+1} \times(r+1)$ binary matrix

$$
\left(\begin{array}{l}
0 G_{0} \\
0 G_{1} \\
0 G_{2} \\
\vdots \\
0 G_{2^{r}-1} \\
1 G_{2^{r}-1} \\
1 G_{2^{r}-2} \\
\vdots \\
1 G_{1} \\
1 G_{0}
\end{array}\right)
$$

Henceforth, we fix $r$ and index the codewords by $0,1, \ldots, 2^{r}-1 \quad\left(\bmod 2^{r}\right)$, denoting the $i$ th codeword in an $r$-bit RGC by $G_{i}$, and the $i$ th codeword in a $k$-bit RGC, where $k \neq r$, by $G_{i}^{(k)}$.

(iii) The flip bit in the $j$ th codeword of a RGC is the position of the one bit that has changed from the $(j-1)$ st codeword.

We will construct an induced subgraph of $X_{n}$ whose residue representation consists of the rows $v_{0}, v_{1}, \ldots, v_{M}$, where $M=2^{r}+1$, and $\left\{v_{i}, v_{j}\right\} \in E$ if and only if $i-j \equiv \pm 1$ $\left(\bmod 2^{r}+2\right)$. Let $v_{M-1} \approx 0100 \cdots 0$, and $v_{M} \approx 122 \cdots 2$. We define the rows $\left\{v_{i}:\right.$ $i$ even, $i \neq M-1\}$ by using the first half of an $r$-bit RGC with a slight modification. Let $\widehat{G}_{i}$, for $i \neq 0$ be the $i$ th codeword $G_{i}$ in an $r$-bit RGC, with the flip bit replaced by a 2 . Let $\widehat{G}_{0}=G_{0}$. Then we define the even-indexed rows as follows: $v_{2 i}=\widehat{G}_{i}$, for $0 \leq i<2^{r-1}$. 


\begin{tabular}{|c|c|c|c|c|}
\hline $\begin{array}{ll}0 & 0\end{array}$ & 0 & 0 & 0 & 0 \\
\hline $\begin{array}{ll}1 & 1\end{array}$ & 1 & 1 & 1 & 1 \\
\hline $\begin{array}{ll}0 & 0\end{array}$ & 2 & 0 & 0 & 0 \\
\hline 11 & 0 & 1 & 1 & 1 \\
\hline 02 & 1 & 0 & 0 & 2 \\
\hline 10 & 0 & 1 & 1 & 0 \\
\hline $\begin{array}{ll}0 & 1\end{array}$ & 2 & 0 & 0 & 1 \\
\hline 10 & 1 & 1 & 1 & 0 \\
\hline $\begin{array}{ll}0 & 1\end{array}$ & 0 & 0 & 2 & 1 \\
\hline 12 & 2 & 1 & 0 & 0 \\
\hline & & 0 & 1 & 1 \\
\hline & & 1 & 0 & 0 \\
\hline & & 0 & 1 & 2 \\
\hline & & 1 & 0 & 1 \\
\hline & & 0 & 1 & 0 \\
\hline & & 1 & 0 & 1 \\
\hline & & 0 & 1 & 0 \\
\hline & & 1 & 2 & 2 \\
\hline
\end{tabular}

Figure 2: We construct two cycles using residue representation and our lower bound construction. On the left is an induced 10-cycle for the graph $X_{n}$, where $n$ has three prime divisors $(r=3)$. On the right is an induced 18-cycle for the graph $X_{n}$, where $n$ has four prime divisors $(r=4)$. Note that the rows in both cycles are derived as described from a 3-bit Reflective Gray Code and a 4-bit Reflective Gray Code, respectively.

We define the odd-indexed rows as follows: for $0 \leq i \leq 2^{r-1}$, let $v_{2 i+1}=\overline{G_{i}}$, the complement of $G_{i}$. So the subgraph we have constructed is

$$
\left\{\widehat{G}_{0}, \bar{G}_{0}, \widehat{G}_{1}, \ldots, \widehat{G}_{2^{r-1}-1}, \bar{G}_{2^{r-1}-1}, v_{M-1}, v_{M}\right\} .
$$

This gives us a subgraph consisting of $\left(2^{r}+2\right)$ vertices.

In Figure 2, we display this construction for $r=3$ and $r=4$.

To prove Theorem 3.1, we must show that the subgraph we have constructed is indeed an induced cycle. This can be reduced to showing that the following properties hold.

(i) Vertex $v_{k}$ is adjacent to $v_{l}$ if $k-l \equiv \pm 1\left(\bmod 2^{r}+2\right)$. In other words, $\left\{v_{0}, v_{1}, \ldots v_{M}\right\}$ is a cycle.

(ii) If neither $k$ nor $l$ equals $M-1$ or $M$, and $|k-l|>1$, then $v_{k}$ is not adjacent to $v_{l}$.

(iii) Vertex $v_{M}$ is not adjacent to $v_{l}$ for $i \neq 0, M-1$, and vertex $v_{M-1}$ is not adjacent to $v_{l}$ for $i \neq M-2, M$.

Proof of Theorem 3.1.

(i) First we show that any two consecutive rows among $v_{0}, v_{1}, \ldots, v_{M-2}$ correspond to adjacent vertices. Among these rows, no odd-indexed row contains a 2, and an evenindexed row $v_{2 i}$ is merely the complement of $v_{2 i+1}$ with one bit replaced by a 2 . Thus every 
odd-indexed row among $v_{0}, v_{1}, \ldots, v_{M-2}$ has no coincidences with the row immediately above it. Also, since any two consecutive codewords $G_{i}$ and $G_{i+1}$ in an $r$-bit RGC differ only in the flip bit of $G_{i+1}$, the codeword $\bar{G}_{i}$ differs from $G_{i+1}$ everywhere except in the flip bit. However, in modifying $G_{i}$ to $\widehat{G}_{i}$ for $0 \leq i<2^{r-1}$, we have replaced every flip bit by a 2 , so $v_{2 i+1}=\bar{G}_{i}$, (which will contain no 2 's), will differ completely from $v_{2 i+2}=\widehat{G}_{i+1}$ if $i \neq 2^{r-1}-1$. Thus every odd-indexed row among $v_{0}, v_{1}, \ldots, v_{M-4}$ is adjacent to the row immediately below it.

It remains to show that $v_{M}$ is adjacent to $v_{M-1}$, that $v_{M}$ is adjacent to $v_{0}$ (these two claims are trivial by inspection), and that $v_{M-2}$ is adjacent to $v_{M-1}$. Note that $v_{M-1}$ is precisely $G_{2^{r-1}-1}$, since, by definition,

$$
G_{2^{r-1}-1}=0 G_{2^{r-2}-1}^{(r-1)}=01 G_{0}^{(r-2)}=0100 \cdots 0 .
$$

Also, $v_{M-2}$ is, by definition, $\bar{G}_{2^{r-1}-1}$. Thus, indeed, $v_{M-2}$ is adjacent to $v_{M-1}$, and we have that $\left\{v_{0}, v_{1}, \ldots v_{M}\right\}$ is a cycle.

(ii) It is trivial to show that no two rows whose indices have the same parity are adjacent, since all even-indexed rows begin with a 0 and are thus not adjacent to each other, while all odd-indexed rows begin with a 1 and are also not adjacent to each other.

Now, take an even-indexed row $v_{2 i}$, with $0 \leq i<2^{r-1}$, and an odd-indexed row $v_{2 j+1}$, with $0 \leq j<2^{r-1}$, such that $i \neq j$ and $i \neq j+1$. Suppose for the sake of contradiction that $v_{2 i}$ is adjacent to $v_{2 j+1}$.

By definition, $v_{2 j+1}=\bar{G}_{j}, v_{2 i}=\widehat{G}_{i}$, and $i \neq j$ by assumption. By the definition of a RGC, $G_{j}$ and $G_{i}$ differ in at least one bit. Since $i-j \not \equiv 1\left(\bmod 2^{r}\right)$, then $G_{j}$ and $G_{i}$ must differ in a bit that is not a flip bit for $G_{i}$. Therefore $v_{2 j+1}=\bar{G}_{j}$ will have at least one coincidence with $v_{2 i}=\widehat{G}_{i}$, and so $v_{2 i}$ and $v_{2 j+1}$ are not adjacent, contrary to our supposition.

So, indeed, if neither $k$ nor $l$ equals $M-1$ or $M$, and $|k-l|>1$, then $v_{k}$ is not adjacent to $v_{l}$.

(iii) Since $v_{M}$ begins with a 1 , it is not adjacent to any of the odd-indexed rows, which also all begin with a 1 . Similarly, because all of the even-indexed rows except $v_{0}$ and $v_{M-1}$ have a 2 in some spot after the initial 0 , and will thus have a coincidence with $v_{M} \approx 122 \cdots 2$, no even-indexed row except $v_{0}$ and $v_{M-1}$ will be adjacent to $v_{M}$.

Since $v_{M-1}$ begins with a 0 , it is not adjacent to any of the even-indexed rows, which all begin with a 0 as well. Also, note that $v_{M-2}=v_{2^{r}-1}=\bar{G}_{2^{r-1}-1}=1011 \cdots 1$ is the complement of $v_{M-1}$, and that all odd-indexed rows except $v_{M}$ are distinct and contain only 0's and 1's. Thus all odd-indexed rows except $v_{M}$ either complement or have a coincidence with $V_{M-1}=0100 \cdots 0$. So all odd-indexed rows except for $v_{M-2}$ and $v_{M}$ are not adjacent to $v_{M-1}$.

Thus we have that vertex $v_{M}$ is not adjacent to $v_{i}$ for $i \neq 0, M-1$, and vertex $v_{M-1}$ is not adjacent to $v_{i}$ for $i \neq M-2, M$.

Note that, for any $n=p_{1} p_{2} \cdots p_{r}$, where $p_{1}<p_{2}<\cdots<p_{r}$ are primes, the cycle constructed above does not depend on the choice of $p_{i}$. The first column of the cycle's 
residue representation contains residues 0 and 1 only, allowing for $p_{1}=2$, and the residue set of the cycle is $\{0,1,2\}$, which puts no bounds on the rest of the primes $p_{i}$.

Also, Theorem 2.10 implies that our construction of a $\left(2^{r}+2\right)$-cycle for $n^{\prime}=p_{1} p_{2} \ldots p_{r}$, $r>1$ holds for $n=p_{1}{ }^{a_{1}} p_{2}{ }^{a_{2}} \ldots p_{r}{ }^{a_{r}}$, while Proposition 2.11 implies that the lower bound in Theorem 3.1 holds for $r=1$.

\section{Generalizing to Other Graphs}

A natural question to ask is what properties of the circulant graph $X_{n}$ are necessary to obtain the results we have. It is noted in [1] that, for $p$ prime and $a$ a positive integer, $X_{p^{a}}$ is complete $p$-partite. In fact, this tells us that for $n=p_{1}^{a_{1}} p_{2}^{a_{2}} \cdots p_{r}^{a_{r}}, X_{n}$ is the conjunction $X_{p_{1}^{a_{1}}} \wedge X_{p_{2}^{a_{2}}} \wedge \cdots \wedge X_{p_{r}^{a_{r}}}$ of graphs $X_{p_{1}^{a_{1}}}, X_{p_{2}^{a_{2}}}, \cdots, X_{p_{r}^{a_{r}}}$, where a conjunction of graphs is defined as follows:

Definition 4.1. Let the graph $G_{1}$ have vertex set $V\left(G_{1}\right)$ and edge set $E\left(G_{1}\right)$, and graph $G_{2}$ have vertex set $V\left(G_{2}\right)$ and edge set $E\left(G_{2}\right)$. Then the conjunction $G_{1} \wedge G_{2}$ has vertex set $V\left(G_{1} \wedge G_{2}\right)=V\left(G_{1}\right) \times V\left(G_{2}\right)$, and $\left(v_{1}, v_{2}\right)$ is adjacent to $\left(u_{1}, u_{2}\right)$ if $v_{1} u_{1} \in E\left(G_{1}\right)$, and $v_{2} u_{2} \in E\left(G_{2}\right)$.

Interestingly, our results can be extended to any conjunction $G_{1} \wedge G_{2} \wedge \cdots \wedge G_{r}$, where each $G_{i}$ is complete $k_{i}$-partite. Let $S=\left\{k_{1}, k_{2}, \ldots, k_{r}\right\}$ be an $r$-tuple of positive integers. Let $\mathcal{G}^{S}=\left\{G \mid G=G_{1} \wedge G_{2} \wedge \cdots \wedge G_{r}\right\}$, where $G_{i}$ is a complete $k_{i}$-partite graph. Denote the length of the longest induced cycle in $G \in \mathcal{G}^{S}$ by $\mathcal{M}(S)$, and define $\mu(r)=\max _{S} \mathcal{M}(S)$ to be the length of the longest induced cycle in all graphs in $\mathcal{G}^{S}$, where $S$ contains $r$ integers.

Theorem 4.2. For $r>1$, we have that $\mu(r)=M(r)$.

To prove Theorem 4.2, we will create for conjunctions of $k_{i}$-partite graphs a representation similar to residue representation. Then, using this representation, we will show how cycles in $G \in \mathcal{G}^{S}$ and $X_{n}$ are related.

Definition 4.3. Let $S=\left\{k_{1}, k_{2}, \ldots, k_{r}\right\}$, and let $G \in \mathcal{G}^{S}, G=G_{1} \wedge G_{2} \wedge \cdots \wedge G_{r}$. Label the partitions in $G_{i}$ by $\left\{0,1,2, \ldots, k_{i}-1\right\}$. Let $v=\left(v_{1}, v_{2}, \ldots, v_{r}\right) \in V(G)$, where $v_{i}$ belongs to partition $\alpha_{i}$ in $G_{i}$. Then the partition representation of $v$ is $\alpha_{1} \alpha_{2} \cdots \alpha_{r}$, and we say $v \simeq \alpha_{1} \alpha_{2} \cdots \alpha_{r}$.

We can define the partition representation of a subgraph of $G \in \mathcal{G}^{S}$ as we defined the residue representation of a subgraph of $X_{n}$. Namely, an induced subgraph on $\left\{x_{1}, x_{2}, \ldots, x_{l}\right\}$ is written as an array of partition representations of the vertices $x_{i}$. Note that an induced subgraph in $G$ is a cycle precisely when its partition representation satisfies the conditions needed for the residue representation of an induced cycle in $X_{n}-$ no two non-consecutive rows can have coincidences, and two non-consecutive rows must have at least one coincidence.

Proof of Theorem 4.2. 
(1) First we show that $M(r) \geq \mu(r)$. Suppose $S=\left\{k_{1}, k_{2}, \ldots, k_{r}\right\}$, and $G \in \mathcal{G}^{S}$ contains an induced cycle $C$ of length $\mu(r)$, whose partition representation is

$\begin{array}{cccc}\alpha_{11} & \alpha_{12} & \cdots & \alpha_{1 r} \\ \alpha_{21} & \alpha_{22} & \cdots & \alpha_{2 r} \\ \vdots & \vdots & & \vdots \\ \alpha_{\mu(r) 1} & \alpha_{\mu(r) 2} & \cdots & \alpha_{\mu(r) r}\end{array}$.

Note that, applying Proposition 2.4 to partition representations, no two rows above are identical if $\mu(r)>4$. So, if $\mu(r)>4$, let $n=p_{1} p_{2} \cdots p_{r}$, where the $p_{i}$ are prime and $p_{i} \geq \max \left\{\alpha_{1 i}, \alpha_{2 i}, \ldots, \alpha_{\mu(r) i}\right\}$. Then the partition representation of $C$ above is in fact also the residue representation of an induced cycle in $X_{n}$, and so $X_{n}$ contains a cycle of length $\mu(r)$, as desired. If $\mu(r) \leq 4$, we know that $M(r) \geq \mu(r)$, since $M(1)=4$, and $M(r)$ increases with $r$ by Proposition 2.8.

(2) Now we show that $\mu(r) \geq M(r)$. Let $X_{n}$, where $n=p_{1} p_{2} \cdots p_{r}$, contain an induced cycle of length $M(r)$. Then $X_{n} \in G^{\left\{p_{1}, \ldots, p_{r}\right\}}$, so $\mu(r) \geq M(r)$, as desired.

Since our original problem concerns the circulant graph $X_{n}$, we are also interested in circulant graphs to which our results generalize. In particular, we are interested in those graphs $G=\operatorname{Cay}\left(A ; A^{*}\right)$, where $A$ is a ring, $A^{*}$ is the group of units in $A$, and the graph $G$ is defined as follows:

(1) The vertex set $V(G)$ of $G$ is the set of elements in $A$.

(2) If $x, y \in V(G)$ then $\{x, y\} \in E(G)$, the edge set of $G$, if and only if $x-y \in A^{*}$.

We know that we can extend our results to any graph $G$ that is a conjunction of complete $k_{i}$-partite graphs for some $k_{i}$. Note that, surprisingly, $k_{i}$ need not be finite, and, in fact, our circulant graph need not contain a finite number of vertices. For this, we rely on a partition using the Chinese Remainder Theorem. One can refer to an algebra text such as [2], pp. 92-97 for the basic facts about rings and ideals needed to prove when such a partition gives us the desired graph structure.

Definition 4.4. A local ring is a ring that contains only one maximal ideal.

With this definition, we can show that a unitary circulant graph on a product of local rings is a conjunction of complete $k_{i}$-partite graphs.

Theorem 4.5. Let $A_{1}, A_{2}, \ldots A_{r}$ be local rings, and let $\mathfrak{m}_{i}$ be the one maximal ideal in $A_{i}$. If $A=A_{1} \times A_{2} \times \cdots \times A_{r}$, then the circulant graph $C a y\left(A ; A^{*}\right)$ is a conjunction of complete $k_{i}$-partite graphs, for some nonzero $k_{i}$.

Theorem 4.5 lets us extend our results to various unitary circulant graphs. In particular, it allows us to generalize to unitary circulant graphs on Dedekind rings.

Definition 4.6. A Dedekind domain ([3]) is an integral domain $R$ such that

(1) Every ideal in $R$ is finitely generated;

(2) Every nonzero prime ideal is a maximal ideal;

(3) $R$ is integrally closed in its field of fractions

$$
K=\{\alpha / \beta: \alpha, \beta \in R, \beta \neq 0\} .
$$


A Dedekind ring is simply a quotient of a Dedekind domain.

If $R$ is a Dedekind domain, and $\mathfrak{m}_{i}$ is a maximal ideal of $R$, then $R / \mathfrak{m}_{i}$ is a field and thus contains only one maximal ideal, (0), and $R / \mathfrak{m}_{i}^{a_{i}}$ contains only the maximal ideal $\mathfrak{m}_{i}$, so $R / \mathfrak{m}_{i}^{a_{i}}$ is a local ring. This is essential for the following corollary.

Corollary 4.7. Let $R$ be a Dedekind domain and let $I=\mathfrak{m}_{1}^{a_{1}} \mathfrak{m}_{2}^{a_{2}} \cdots \mathfrak{m}_{r}^{a_{r}}$ be a nonzero, nonunit ideal in $R$, where $\mathfrak{m}_{i}$ are maximal ideals of $R$. Then the circulant graph Cay $\left(A ; A^{*}\right)$ is a conjunction of complete $k_{i}$-partite graphs, for $k_{i}=\#\left(R / \mathfrak{m}_{i}\right)$.

Proof. Since $\mathfrak{m}_{i}$ are the distinct maximal ideals, $\mathfrak{m}_{i}^{a_{i}}+\mathfrak{m}_{j}^{a_{j}}=R$ for all $1 \leq i<j \leq r$. Then the Chinese Remainder Theorem implies that

$$
A=R / \mathfrak{m}_{1}^{a_{1}} \mathfrak{m}_{2}^{a_{2}} \cdots \mathfrak{m}_{r}^{a_{r}}=R / \mathfrak{m}_{1}^{a_{1}} \times R / \mathfrak{m}_{2}^{a_{2}} \times \cdots \times R / \mathfrak{m}_{r}^{a_{r}}
$$

We have noted above that $R / \mathfrak{m}_{1}^{a_{1}}$ is local, and thus we have that $A$ is a product of local rings. By Theorem 4.5, we have that the circulant graph $\operatorname{Cay}\left(A ; A^{*}\right)$ is a conjunction of complete $k_{i}$-partite graphs, for $k_{i}=\#\left(R / \mathfrak{m}_{i}\right)$.

So, indeed, our theorems concerning $M(r)$ generalize to the maximum lengh of a cycle in unit circulant graphs on a Dedekind domain quotiented by an ideal with $r$ distinct maximal factors. Dedekind domains are exactly those integral domains in which every ideal has a unique factorization into prime ideals, and thus are the rings of number theoretical interest. Some nice examples of the Dedekind rings that we have generalized to above are the Gaussian integers modulo $a+b i$, denoted by $\mathbb{Z}[i] /(a+b i)$; any quotient of the ring of algebraic integers in the $p$ th cyclotomic field $\mathbb{Z}\left[\zeta_{p}\right]$, where $\zeta_{p}$ is a $p$ th root of unity; and any quotient of $\mathbb{C}[x, y] /\left(y^{2}-x^{3}+x\right)$, the ring of regular functions on the elliptic curve $y^{2}=x^{3}-x$. Note that we also have generalized to unit circulant graphs on quotients of principal rings.

\section{Acknowledgements}

This research was done at the University of Minnesota Duluth. I would like to give special thanks to Joseph A. Gallian for his encouragement and support, and I would like to thank Philip Matchett, Melanie Wood, and Justin Sinz for their insightful comments. I would also like to thank Geir Helleloid for helpful conversations. Funding was provided by grants form the NSF (DMS-0137611) and NSA (H-98230-04-1-0050).

\section{References}

[1] P. Berrizbeitia, R. E. Giudici, On cycles in the sequence of unitary Cayley graphs, Discrete Math 282 (2004), 1-3.

[2] S. Lang. Algebra, 3rd edition, Springer-Verlag, NY, 2002.

[3] D. A. Marcus. Number Fields, Springer Verlag, NY, 1977.

[4] E. Reingold, J. Nievergelt, N. Deo. Combinatorial Algorithms, Prentice Hall, NJ, 1977. 